\title{
Competencias del Profesorado de la Escuela Rural Catalana para Abordar la Dimensión Territorial en el Aula Multigrado
}

\section{Catalan Rural Schools Teachers' Skills to Face the Territorial Dimension in the Multigrade Classroom}

\author{
Roser Boix * \\ Francesc Buscà
}

Universitat de Barcelona, España

\begin{abstract}
En este artículo se presentan los resultados parciales de un estudio descriptivo realizado en el marco del proyecto FOPROMAR (2017-1-ESo1-KA201-038217). En concreto, se muestran los resultados obtenidos tras la administración del Cuestionario de Competencias del Profesorado de la Escuela Rural (CC-PER) a los maestros y maestras que ejercen su labor docente en las escuelas rurales de Cataluña $(\mathrm{n}=276)$. Además de los datos de contexto, este cuestionario se compone de 30 competencias agrupadas en los siguientes ámbitos: competencias pedagógicas, competencias metodológicas y competencias de relación escuela-territorio. Su propósito es que el profesorado valore la relevancia y el grado de dominio de las competencias relacionadas con la dimensión territorial. El análisis de los datos se ha realizado teniendo en cuenta los estadísticos descriptivos básicos (media, mediana, desviación estándar y varianza), y el cálculo del índice Alpha de Cronbach para comprobar el grado de consistencia interna de las puntuaciones otorgadas en cada ámbito competencial. Los principales resultados indican que: las competencias seleccionadas son pertinentes y representativas de la dimensión territorial; el grado de dominio de estas competencias suele ser peor valorado que cuando se valora su relevancia; y que estas competencias peor valoradas deberían ser tenidas en cuenta para el diseño de planes específicos de formación.
\end{abstract}

Descriptores: Educación básica; Educación rural; Competencia profesional; Competencias del docente; Formación de docentes.

This paper presents the partial results that gathered in a descriptive study made inside the FOPROMAR Project (2017-1-ESO1-KA201-038217). It shows the results from the Questionnaire Teaching Skills' Rural School Teachers (CC-PER) passed to Catalan rural school's teachers $(\mathrm{n}=276)$. Furthermore, background data, this questionnaire had 30 competences classified in these areas: pedagogical competences; methodological competences and relationship school-territory competences. The goal of this questionnaire was to evaluate the value and the master of the teaching skills in the territorial dimension by the rural school teachers who participated in this study. The descriptive statistics (mean; standard deviation and the variance) were the basis of the data analysis. In addition, the Cronbach Alpha index showed the consistency between the score obtained to every competence area. The main results indicate that skills selected and included in this questionnaire are useful to evaluate the territorial dimension; the mastering of this skills are less appreciating than the grade of value of these skills; and finally, it's necessary these differences should be considered to design specific teaching degrees or master degrees.

Keywords: Basic education; Rural education; Occupational qualifications; Teaching profession; Teacher education schools.

*Contacto: roser.boix@ub.edu

ISSN: 1696-4713

www.rinace.net/reice/

revistas.uam.es/reice
Recibido: $\quad 1$ de octubre 2019

$1^{\text {a }}$ Evaluación: 13 de enero 2020

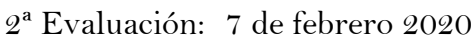

Aceptado: 16 de febrero 2020 


\section{Introducción}

El dominio de las competencias profesionales relacionadas con la dimensión territorial es un aspecto clave para el profesorado que quiere desempeñar su labor docente en la escuela rural.

Este ámbito competencial es fundamental para el diseño, la implementación y la evaluación de proyectos educativos y propuestas didácticas vinculadas significativamente con el territorio en que se sitúa la escuela. Se trata de competencias que capacitan al profesorado de la escuela rural para la adquisición de saberes y valores que constituyen el patrimonio y capital cultural del territorio al cual pertenece la escuela. Y también para el dominio de estrategias y habilidades que le permiten relacionarse con los agentes internos y externos que forman parte de la comunidad educativa y el territorio rural, e implicarlos en el proyecto educativo de la escuela.

Sin embargo, el desempeño de estas competencias profesionales específicas suele estar condicionado por estas problemáticas:

- La dicotomía escuela rural y escuela urbana: si bien se parte del mismo marco curricular de referencia, educar en la escuela rural trae consigo asumir un enfoque epistemológico y metodológico que difiere del modelo de escuela urbana. Por tanto, ejercer como docente en la escuela rural implica adoptar una función y unos roles diferentes a los adoptados en la escuela urbana (Boix, 2014; Chaparro-Aguado y Rubio, 2010; Cornish, 2006). Entre otros aspectos, ser maestro o maestra en la escuela rural supone aceptar que los proyectos educativos tengan como referencia las necesidades y los retos sociales y educativos vinculados a la ruralidad; optar tanto por una organización del aula multigraduada como por una metodología eminentemente activa y participativa; $\mathrm{y}$, sobre todo, formar parte e implicarse en la vida del municipio.

- Falta de consolidación de proyectos educativos sensibles con las necesidades y problemas del territorio: el profesorado no suele optar por ejercer su labor en la escuela rural. Por norma general, su prioridad es integrarse en escuelas pertenecientes a áreas urbanas o a poblaciones mayores a 1.500 habitantes por m² (Abós, 2011; Bustos, 2011; Domingo, 2014, Solé, 2016). En el caso de ser destinado a una escuela rural, y a pesar de que el profesorado suele valorar positivamente la experiencia, intenta cambiar de destino lo antes posible, en busca del destino deseado. Este hecho puede explicar por qué el profesorado difícilmente contempla la posibilidad de planificar un proyecto de vida a medio o largo plazo en estas poblaciones y, por extensión, que las plantillas de estas escuelas rurales carezcan del arraigo y el sentido de pertenencia al territorio necesario para implementar propuestas educativas acordes con las necesidades del entorno.

- Formación inicial y continuada específica escasa: las facultades de formación del profesorado no suelen tener presente una formación específica centrada en la escuela rural (Abós, 2015; Arriaga y Ruiz-Gallardo, 2017; Raso, Hinojo y Solá, 2015). Si bien este tópico suele ser tenido en cuenta en las prácticas curriculares, como contenido en asignaturas de corte didáctico o, incluso en cursos de formación continuada, su tratamiento suele ser tangencial y, por tanto, no suele profundizar en sus problemáticas y retos educativos actuales. 
Teniendo como referencia esta problemática, el proyecto "La Formación profesional y las competencias del maestro rural como dinamizador de la dimensión territorial en la escuela (FOPROMAR)" tiene como finalidad principal proponer un plan de formación permanente y unas propuestas de formación inicial para mejorar las competencias profesionales del profesorado que ejerce su labor docente en escuelas rurales de las regiones pertenecientes a los siguientes países: Portugal (Alto Alentejo), España (Catalunya y Aragón), y Francia (Aquitania).

En concreto, los objetivos específicos de este proyecto son los siguientes:

- Identificar las competencias del docente rural para llevar a cabo su función formativa y las competencias que tiene como dinamizador de la dimensión territorial de la escuela rural.

- Determinar los saberes educativos latentes en la escuela y/o en el territorio que permiten impulsar desde la escuela la dimensión territorial.

- Elaborar las bases que orienten el diseño de planes de formación inicial y permanente para los docentes rurales y presentación pública del proyecto en cada estado socio.

Este artículo se centra en los resultados específicos obtenidos en la Comunidad Autónoma de Cataluña (España). En concreto, se presentan los resultados referidos al balance que los maestros y maestras de la escuela rural catalana realizan con respecto a la relevancia y su grado de dominio de las competencias del relacionado con la dimensión territorial.

Por consiguiente, los objetivos del presente trabajo son:

- Definir las características del profesorado y de los contextos en donde desempeña sus competencias relacionadas con la dimensión territorial.

- Identificar las competencias profesionales que el profesorado de la escuela rural catalana considera necesarias para el desempeño de la dimensión territorial en el aula.

- Constatar el balance que el profesorado realiza con respecto al grado de dominio de estas competencias.

Sobre la base de estos objetivos, el fin último es disponer de evidencias pertinentes y relevantes que permitan el diseño fundamentado de un plan de formación permanente y unas propuestas de formación inicial para cubrir las necesidades competenciales de los maestros y maestras rurales de Catalunya.

\section{La escuela rural en Cataluña: Ruralidad y multigraduación}

En Cataluña, el territorio rural ha sufrido grandes transformaciones en las últimas décadas. Este hecho ha acarreado cambios significativos en las formas de vida, y en el propio uso rural del territorio. Uno de los cambios más significativos ha sido que la dicotomía entre campo y ciudad, entre entorno rural y urbano se haya hecho cada vez más invisible. Tal y como se indica en el Atles de la Nova Ruralitat, esto ha supuesto que:

La referencia a la actividad y a las formas de vida que estaban vinculadas a la ruralidad dejan paso a apreciaciones estrictamente demográficas o de tipo más 
sociológico como la densidad demográfica, el nombre de habitantes, y a otros más cualitativos como la presencia de comercios, servicios, accesibilidad. (Serret y Trepat, 2009, p.11)

Los territorios rurales se concentran en las zonas interiores. En estas áreas se encuentran las comarcas que apenas alcanzan los 20.00-30.000 habitantes y que coinciden con las más alejadas de la zona metropolitana de Barcelona; éstas son las que presentan condiciones más rurales. A ellas hay que sumarle la población diseminada que se considera el hábitat rural por excelencia; la población diseminada es aquella que "no forman una agrupación superior a 9 viviendas y se sitúan a una distancia mínima superior a los 200 metros del núcleo de población” (Serret y Trepat, 2009, p. 23); en Cataluña la población diseminada es relevante, y abarca desde las áreas pre-pirenaicas hasta la zona de la Horta de Lleida y las urbanizaciones de la Cerdaña y las próximas a la costa.

En la actualidad el territorio rural catalán tiene un notable nivel de satisfacción en cuanto a la generalización de la educación infantil y primaria. Además, el acceso a internet, junto con la presencia de equipamientos deportivos, culturales básicos y, por supuesto, de servicios básicos como agua, electricidad, recogida de basura son, entre otros, realidades presentes en los pequeños municipios o núcleos de población rural, con muy pocas excepciones. Todo ello ha mejorado sustancialmente la calidad de la vida en el territorio rural (Trepat, 2015).

En Cataluña existen dos tipologías básicas de escuela rural que han facilitado la generalización de la educación infantil y primaria: la unitaria (una o dos clases) y la cíclica (más de dos clases y menos de ocho). Ambas tipologías se caracterizan por acoger en sus aulas la mutigraduación, por lo que no se entiende la escuela rural sin la existencia de alumnos de distintas edades que comparten una misma clase.

Estos centros escolares (unitarios y/o cíclicos) se agrupan en las llamadas Zonas Escolares Rural (ZER). Una ZER es un grupo de escuelas rurales que comparten un proyecto educativo y maestros especialistas itinerantes. Una ZER tiene sus propios órganos de gobierno unipersonales y colegiados. No todas las escuelas rurales se agrupan en ZER, pero sí disponen de maestros especialistas itinerantes como las agrupadas en ZER. Los maestros especialistas se desplazan a lo largo de las escuelas que conforman agrupación e imparten su especialidad en Educación Musical, Educación Física y Lengua Extranjera, básicamente.

En los últimos años, la política educativa catalana ha impulsado la escuela rural. Si bien las ZER nacen a principios de los años 80, se han mantenido hasta la actualidad, dando una identidad propia a la educación rural; cada escuela, por muy pequeña que sea, mantiene sus órganos básicos de gobierno unipersonales y colegiados. Ello ha facilitado que cada centro escolar, a pesar de formar parte de un agrupamiento, pueda mantener su autogestión territorial, cultural y curricular.

Los maestros rurales han realizado un gran trabajo en las escuelas; a pesar de una escasa formación inicial y permanente, como veremos a continuación, han sido capaces de mantener una institución escolar arraigada al territorio y con niveles de calidad equiparables a los urbanos (Buscà, Domingo y Boix, 2018). Además, el trabajo pedagógico realizado por el Secretariado de Escuela Rural y otras asociaciones de maestros, junto con la indagación constante de grupos de investigadores de la Universidades Catalanas y la Federación del Mundo Rural Catalán, ha permitido avanzar en la mejorar de la calidad de la educación en las escuelas rurales catalanas. 


\section{Dimensión territorial, escuela rural y formación del maestro rural}

La dimensión territorial aporta una visión interdisciplinar (cultural, social, espacial, económica, política) del ámbito rural, en la cual prevalece un sistema de interacciones sociales y organizadas entre sí que junto con instituciones estructuradas de carácter público y/o privado fortalecen las capacidades de los principales actores educativos rurales. La escuela es uno de estos actores, de hecho, su principal actor educativo, y como tal juega un papel clave en la progresiva transformación que viene sufriendo el territorio rural.

Los adagios de que "sin escuela no hay pueblo", o que "el pueblo se muere sino hay escuela" no solo reflejan ese papel activo e indispensable que la escuela tiene en el territorio, sino que muestran también el protagonismo de una institución escolar que además de ejercer las funciones propias de esta institución, su acometividad repercute en la estructura de interacciones sociales y organizadas como miembro activo del sistema institucional territorial, como receptora de identidades y emociones, como capital social local y como elemento configurador en la construcción social rural (Boix, 2014).

Estas atribuciones y tareas que desempeña la escuela rural en el marco de la dimensión territorial donde se encuentra ubicada, no significa que realmente tenga las capacidades necesarias para ello, y aún más, tampoco presupone que tenga la percepción de que así sea; los maestros junto con la comunidad educativa y la propia comunidad rural son los agentes de los que se espera que puedan seguir arraigando la escuela en el pueblo en función de las responsabilidades que tienen cada uno de ellos; sin embargo, los maestros no tienen, en general, las competencias profesionales básicas para poder emprender este cometido.

La escuela rural y la dimensión territorial rural no forman parte, en general, del contenido de los planes de estudio de los Grados de Maestro. Algunas instituciones universitarias del Estado Español tratan el tema en ciertas asignaturas obligatorias o en asignaturas optativas; incluso, se ofrece a los estudiantes la posibilidad de realizar sus prácticas externas en estos contextos; pero no es en todas las Facultades de Educación ni es suficiente lo que se trata en las que sí que contemplan la escuela rural en la planificación curricular; en los planes de formación inicial debería considerarse la escuela rural, como se atiende la escuela urbana de manera que los futuros maestros conocieran como es la práctica pedagógica con grupos multigrado, además de la articulación de las interacciones sociales de las comunidades rurales; la consecución de competencias profesionales para ejercer en una escuela multigrado así como el de conocer saberes relacionados con el espacio rural debería ser uno de los objetivos principales en los planes docentes de los grados de Maestro.

Si bien en la formación inicial las competencias profesiones y los saberes para ejercer de maestro en una escuela rural son escasas, tampoco son alentadoras las capacidades y competencias que se proyectan en la formación permanente; la mayor parte de los planes de desarrollo profesional van dirigidos a maestros que trabajan en escuelas ordinarias, olvidando las necesidades didáctico-formativas de los docentes de escuela multigrado; no puede ser "adaptada" la instrucción diseñada para escuelas graduadas a las escuelas unitarias y/o cíclicas; no sirve de mucho que un maestro multigrado deba transformar la capacitación que recibe desde una perspectiva de aula graduada a un proceso de aprendizaje multigrado. Es necesario diseñar e implementar planes de formación permanente con contenidos y modalidades formativas programados para la escuela rural, 
y proyectados para la consolidación de proyectos educativos sensibles y adecuados al territorio donde se encuentra emplazada la escuela.

Esta formación inicial y permanente permitiría fortalecer y consolidar la escuela rural como ese actor dinámico y activo que es, e impulsaría la construcción de procesos de desarrollo endógeno de carácter educativo y social, necesarios en los territorios rurales, pero tan subestimados todavía por las políticas educativas y las instituciones de estudios superiores.

\section{Método}

\section{Enfoque metodológico}

Este estudio de naturaleza descriptiva se corresponde con un diseño de investigación no experimental basado en la administración de un cuestionario de encuesta (McMillan y Shumacher, 2011).

\section{Participantes}

La muestra de este estudio se compone de 276 docentes de las escuelas rurales catalanas. Según los datos que dispone el Departament d'Educació de la Generalitat de Catalunya, la población actual de maestros y maestras rurales es de 2.766. Por tanto, puede concluirse que con un margen de error del $5 \%$, la muestra seleccionada es representativa en más de un $90 \%$.

\section{Instrumento}

El Cuestionario Competencias del Profesorado de la Escuela Rural (CC-PER) recoge 30 competencias relacionadas con el desempeño de la dimensión territorial en la escuela rural (ver anexo).

Estas competencias se agrupan en tres ámbitos competenciales:

- Ámbito pedagógico: agrupa 6 competencias clave (ítems del 1 al 6) en las que el profesorado desempeña su capacidad para adecuar su proyecto pedagógico personal a las características y necesidades tanto del entorno como de implicar y colaborar con el resto de docentes para desarrollar proyectos compartidos vinculados al territorio.

- Ámbito metodológico: contiene 17 competencias instrumentales (ítems del 7 al 23) en las que los y las docentes muestran su capacidad para diseñar, gestionar y evaluar proyectos educativos, propuestas didácticas y recursos en las que se tenga en cuenta las problemáticas y retos del territorio y el alumnado.

- Ámbito relación escuela-territorio: agrupa 7 competencias de carácter sistémico (ítems del 24 al 30) en la que el profesorado muestra su capacidad para fomentar implicar y fomentar la participación en los proyectos educativos y las propuestas didácticas de los miembros de la comunidad escolar y los agentes sociales y educativos del territorio.

Tras un proceso previo de documentación teórica, la selección definitiva de estas competencias se lleva a cabo tras consultar su pertinencia con maestros y maestras rurales experimentados, y constatar su consistencia interna tanto para valorar su importancia como el grado de dominio por parte de los encuestados $(\alpha=0,97)$. 
Por otra parte, el cuestionario que se presenta a los participantes en el estudio consta de las siguientes secciones: 1) breve descripción de la investigación y de las instrucciones para su cumplimentación; 2) datos personales y del contexto en el que el profesorado desempeña su labor docente; 3) relación de competencias relacionadas con los ámbitos explicados anteriormente; y 4) apartado en blanco el que el profesorado encuestado propone otras competencias no tenidas en cuenta en el listado.

\section{Procedimiento}

El cuestionario se envía a los maestros y maestras de las escuelas rurales de Catalunya en formato electrónico, a través de la aplicación Google forms.

Teniendo en cuenta las dificultades para acceder in situ a todo el profesorado de las escuelas rurales (generalistas e itinerantes), se emplea un método no probabilístico por bola de nieve. Para ello se utiliza la base de datos del Secretariat d'Escola Rural de Catalunya con la intención de enviar el cuestionario a la dirección personal e institucional de correo electrónico de todos los directores de las escuelas censadas en el Departament d'Educació. Además de responder el cuestionario, los miembros del equipo directivo reciben el encargo de remitirlo al profesorado de su escuela para que éstos puedan reenviarlo cumplimentado a los responsables del estudio.

La persona encuestada debe puntuar por medio de dos escalas de Likert tanto su relevancia para la formación del profesorado de la escuela rural, como el grado en que esta persona considera que domina dicha competencia. En ambos casos, las puntuaciones se corresponden con los siguientes valores: 1 (nada); 2 (reducido); 3 (mediano) y 4 (elevado).

La recepción de cuestionarios se mantiene activa prácticamente durante un año natural, procurando evitar los finales y los inicios de curso y, por tanto, los periodos en que el profesorado suele tener mayores dificultades para responder el cuestionario.

\section{Análisis de datos}

En primer lugar, se calculan los estadísticos descriptivos (media, mediana, desviación estándar y varianza) de las variables vinculadas a los datos de contexto y de las valoraciones de competencias tanto en lo que respecta a su relevancia como al grado de dominio. En este caso también se calcula la diferencia entre las medias obtenidas al valorar el grado de dominio de cada una de las competencias destacadas en el cuestionario y las puntaciones referidas a su relevancia.

En segundo lugar, para comprobar en qué grado las respuestas obtenidas para cada grupo de competencias son consistentes, se calcula también el análisis de consistencia interna (Alfa de Cronbach).

\section{Resultados}

La presentación de resultados se realiza sobre la base de los objetivos de la investigación. Consta de estos tres apartados: descripción del perfil y de las características de los contextos en donde se están desempeñando las competencias vinculadas con la dimensión territorial; la relevancia de estas competencias; y el grado de desempeño de estas competencias por parte del profesorado encuestado. 


\section{1. ¿Cuál es el perfil del profesorado y en que contextos debe desempeñar las competencias vinculadas a la dimensión territorial?}

El perfil del profesorado es un aspecto clave para hacer una prospectiva acerca de las posibilidades de abordar el enfoque curricular basado en competencias en la escuela rural. En el caso que nos ocupa, los datos indican que el perfil tipo que está llevando a cabo este reto es el de una maestra ( $87,3 \%)$ de mediana edad ( $\mathrm{M}=44$ años; $\mathrm{DE} \pm 9,29)$; que cuenta ya con una cierta experiencia en la enseñanza $(M=12,3$ años; $\mathrm{DE} \pm 9,04)$, de los cuales buena parte de ellos se han desarrollado en la escuela rural ( $M=9,34$ años; $\mathrm{DE} \pm 7,43)$.

La mayor parte del profesorado encuestado expresa tener una situación laboral estable (82,2 \% son funcionarios), y su deseo por ejercer como maestro y maestra en una escuela rural $(94,6 \%)$. Por otra parte, en lo que respecta a la formación específica, buena parte del profesorado encuestado manifiesta no haber recibido formación relacionada con la educación en territorios rurales (53,5\%), y tampoco ninguna formación relacionada con el desempeño de competencias profesionales específicas del profesorado de la escuela rural $(77,5 \%)$.

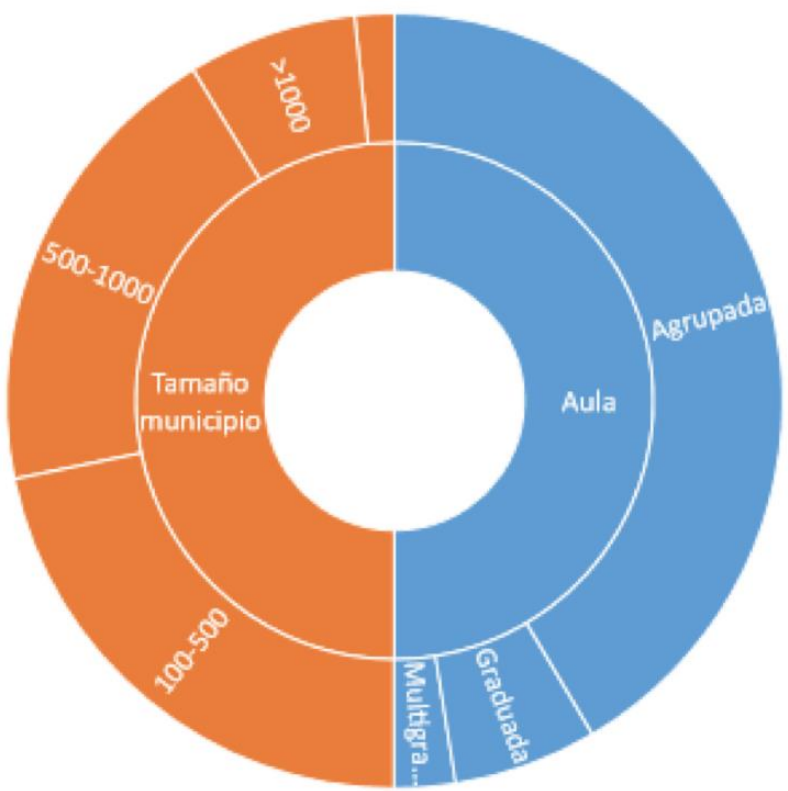

Figura 1. Características del contexto en donde el profesorado de la escuela rural ejerce su labor docente

Fuente: Elaboración propia a partir de los resultados tabulados.

En lo que se refiere al contexto, la figura 1 muestra que el profesorado de la escuela rural ejerce su labor docente en centros organizados mayoritariamente en aulas agrupadas $(83,0 \%)$ y en escuelas pertenecientes a municipios de entre 100 y 500 habitantes $(43,5 \%)$ y 500 y 1000 habitantes $(39,1 \%)$.

\section{2. ¿Qué competencias vinculadas a la dimensión territorial son las más relevantes?}

Los resultados muestran que el profesorado encuestado califica como elevada la necesidad de desempeñar competencias relacionadas con la dimensión territorial (cuadro 1). En este sentido, se observa que las puntuaciones medias oscilan entre un rango que va de los 3,24 a los 3,76 puntos. 
Cuadro 1. Resultados sobre la relevancia de las competencias relacionadas con la dimensión territorial

\begin{tabular}{|c|c|c|c|c|c|}
\hline ÁMBITO & C & $\mathbf{M}$ & MED. & DE & $\Sigma^{2}$ \\
\hline \multirow{6}{*}{ Pedagógico } & 1 & 3,53 & 4 & 0,57 & 0,33 \\
\hline & 2 & 3,61 & 4 & 0,55 & 0,30 \\
\hline & 3 & 3,43 & 4 & 0,69 & 0,47 \\
\hline & 4 & $3,4+1$ & 3 & 0,66 & 0,43 \\
\hline & 5 & 3,59 & 4 & 0,59 & 0,35 \\
\hline & 6 & 3,53 & 4 & 0,61 & 0,37 \\
\hline \multirow{17}{*}{ Metodológico } & 7 & 3,67 & 4 & 0,51 & 0,26 \\
\hline & 8 & 3,59 & 4 & 0,53 & 0,29 \\
\hline & 9 & 3,65 & 4 & 0,54 & 0,29 \\
\hline & 10 & 3,62 & 4 & 0,54 & 0,29 \\
\hline & 11 & 3,61 & 4 & 0,54 & 0,29 \\
\hline & 12 & 3,64 & 4 & 0,52 & 0,27 \\
\hline & 13 & 3,60 & 4 & 0,57 & 0,32 \\
\hline & 14 & 3,66 & 4 & 0,55 & 0,30 \\
\hline & 15 & 3,46 & 4 & 0,63 & 0,40 \\
\hline & 16 & 3,54 & 4 & 0,62 & 0,39 \\
\hline & 17 & 3,76 & 4 & 0,45 & 0,21 \\
\hline & 18 & 3,66 & 4 & 0,53 & 0,29 \\
\hline & 19 & 3,29 & 3 & 0,74 & 0,54 \\
\hline & 20 & 3,37 & 3 & 0,67 & 0,45 \\
\hline & 21 & 3,37 & 3 & 0,67 & 0,45 \\
\hline & 22 & 3,25 & 3 & 0,74 & 0,55 \\
\hline & 23 & 3,32 & 3 & 0,69 & 0,48 \\
\hline \multirow{7}{*}{$\begin{array}{l}\text { Relación } \\
\text { escuela- } \\
\text { comunidad }\end{array}$} & 24 & 3,34 & 3 & 0,71 & 0,51 \\
\hline & 25 & 3,31 & 3 & 0,69 & 0,48 \\
\hline & 26 & 3,47 & 4 & 0,62 & 0,38 \\
\hline & 27 & 3,61 & 4 & 0,55 & 0,30 \\
\hline & 28 & 3,36 & 3 & 0,69 & 0,48 \\
\hline & 29 & 3,24 & 3 & 0,72 & 0,52 \\
\hline & 30 & 3,29 & 3 & 0,75 & 0,56 \\
\hline
\end{tabular}

Nota: Sombreados en gris los resultados más destacados.

Fuente: Elaboración propia a partir de los resultados tabulados.

Por otra parte, el análisis de los datos por cada uno de los ámbitos competenciales identificados en este estudio permite destacar lo siguiente.

\section{Ámbito pedagógico}

Tal y como muestra el análisis de confianza, todas las competencias de este ámbito son pertinentes y muestran un índice elevado de consistencia interna $(\alpha=0,85)$

Si bien todas las competencias de este ámbito se aproximan a la máxima valoración, las competencias 2 y 5 relacionadas con la capacidad del profesorado para incluir en sus proyectos educativos las características culturales del territorio $(\mathrm{M}=3,61, \mathrm{DE}=0,55)$ e implicar a sus compañeros en estos proyectos $(\mathrm{M}=3,59, \mathrm{DE}=0,59)$ son las más 
destacadas en cuanto a su relevancia. El hecho que estas dos competencias presenten una varianza relativamente baja $\left(\sigma^{2}=0,30\right.$ y $\left.\sigma^{2}=0,35\right)$, podría considerarse como un indicio acerca del consenso existente entre el profesorado encuestado acerca de su importancia para la formación del maestro de la escuela rural.

\section{Ámbito metodológico}

Este ámbito, estrechamente relacionado con las competencias profesionales de corte metodológico, es el que presenta un mayor número de competencias a valorar. Los resultados obtenidos tras el análisis de confianza, también muestran que las respuestas referidas a su pertinencia son muy consistentes $(\alpha=0,95)$. Este hecho se refuerza al observar también que se trata del ámbito que, en líneas generales, presenta las puntuaciones más elevadas.

Un análisis más concreto a las competencias de este ámbito permite constatar que las competencias con mayor puntuación son las siguientes:

- C7: Sensibilizar al alumnado sobre las necesidades relacionados con el desarrollo sostenible de su territorio rural $(\mathrm{M}=3,67, \mathrm{DE}=0,51)$.

- C8: Desarrollar el conocimiento de la diversidad cultural de su territorio rural $(\mathrm{M}=3,59, \mathrm{DE}=0,53)$.

- C9: Desarrollar una actitud crítica ante los valores de su territorio rural ( $\mathrm{M}=$ $3,65, \mathrm{DE}=0,54)$.

- C10: Tener en cuenta los conocimientos previos relacionados con la cultura de su territorio rural $(\mathrm{M}=3,62, \mathrm{DE}=0,54)$.

- C11: Establecer relaciones entre las experiencias previas vinculadas a su territorio rural y los nuevos aprendizajes $(\mathrm{M}=3,61, \mathrm{DE}=0,54)$.

- C12: Relacionar los contenidos con la realidad territorial inmediata $(\mathrm{M}=3,64$, $\mathrm{DE}=0,52)$.

- C13: Proponer situaciones de investigación relacionadas con el entorno rural próximo $(\mathrm{M}=3,60, \mathrm{DE}=0,57)$.

- C14: Desarrollar actividades para el conocimiento de la cultura local (M = 3,66, $\mathrm{DE}=0,55)$.

- C17: Utilizar los recursos que ofrece su territorio rural (naturales, materiales, sociales y culturales $)(\mathrm{M}=3,76, \mathrm{DE}=0,45)$.

- C18: Implicar en el centro a agentes de la comunidad local $(\mathrm{M}=3,66, \mathrm{DE}=$ $0,53)$.

Como puede observarse, las valoraciones de todas estas competencias superan los 3,50 puntos, y la varianza que muestran se sitúa en un rango bajo valoraciones $\left(\sigma^{2}=\right.$ entre 0,26 y 0,32 ). Por tanto, también puede decirse que existe un consenso considerable entre el profesorado a la hora de valorar su relevancia.

Ámbito relación escuela-comunidad

Al igual que el resto de ámbitos, gracias al análisis de confianza, puede decirse que todas las competencias de este ámbito sistémico son pertinentes y muestran un índice elevado de consistencia interna $(\alpha=0,87)$. Si bien las valoraciones con respecto a la relevancia de 
todas las competencias de este ámbito se sitúan en un nivel medio-alto (con una puntación media que oscila en un rango que va desde los 3,29 a los 3,61 puntos), las competencias 26 (Comprometerse con iniciativas socio-culturales de la comunidad) y 27 (Establecer procesos de participación escuela-familias-comunidad y viceversa) serían las mejor valoradas $(\mathrm{M}=3,47$ y $\mathrm{M}=3,61$, respectivamente). En este caso, el hecho que las respuestas de estas dos competencias sean las que presentan una menor varianza $\left(\sigma^{2}=\right.$ 0,38 y $\left.\sigma^{2}=0,30\right)$ confirmaría la relevancia de estas valoraciones.

Cuadro 2. Resultados sobre el dominio de las competencias relacionadas con la dimensión territorial

\begin{tabular}{|c|c|c|c|c|c|}
\hline ÁMBITO & C & $\mathbf{M}$ & MED & DE & $\Sigma^{2}$ \\
\hline \multirow{6}{*}{ Profesional } & 1 & 2,95 & 3 & 0,69 & 0,48 \\
\hline & 2 & 3,15 & 3 & 0,67 & 0,45 \\
\hline & 3 & 2,71 & 3 & 0,84 & 0,71 \\
\hline & 4 & 2,68 & 3 & 0,84 & 0,70 \\
\hline & 5 & 3,03 & 3 & 0,77 & 0,59 \\
\hline & 6 & 2,84 & 3 & 0,86 & 0,75 \\
\hline \multirow{17}{*}{ Metodológico } & 7 & 3,05 & 3 & 0,78 & 0,60 \\
\hline & 8 & 3,11 & 3 & 0,73 & 0,53 \\
\hline & 9 & 3,05 & 3 & 0,76 & 0,58 \\
\hline & 10 & 3,14 & 3 & 0,71 & 0,51 \\
\hline & 11 & 3,13 & 3 & 0,69 & 0,48 \\
\hline & 12 & 3,17 & 3 & 0,70 & 0,49 \\
\hline & 13 & 2,95 & 3 & 0,86 & 0,75 \\
\hline & 14 & 3,17 & 3 & 0,72 & 0,52 \\
\hline & 15 & 3,00 & 3 & 0,79 & 0,63 \\
\hline & 16 & 3,03 & 3 & 0,80 & 0,65 \\
\hline & 17 & 3,29 & 3 & 0,70 & 0,49 \\
\hline & 18 & 3,11 & 3 & 0,77 & 0,59 \\
\hline & 19 & 2,49 & 2 & 0,92 & 0,85 \\
\hline & 20 & 2,81 & 3 & 0,84 & 0,70 \\
\hline & 21 & 2,77 & 3 & 0,88 & 0,78 \\
\hline & 22 & 2,71 & 3 & 0,82 & 0,68 \\
\hline & 23 & 2,71 & 3 & 0,82 & 0,67 \\
\hline \multirow{7}{*}{$\begin{array}{l}\text { Relación } \\
\text { escuela- } \\
\text { comunidad }\end{array}$} & 24 & 2,66 & 3 & 0,91 & 0,82 \\
\hline & 25 & 2,61 & 3 & 0,87 & 0,76 \\
\hline & 26 & 2,96 & 3 & 0,80 & 0,64 \\
\hline & 27 & 3,12 & 3 & 0,77 & 0,59 \\
\hline & 28 & 2,96 & 3 & 0,82 & 0,68 \\
\hline & 29 & 2,71 & 3 & 0,85 & 0,72 \\
\hline & 30 & 2,77 & 3 & 0,89 & 0,80 \\
\hline
\end{tabular}

Nota: Sombreados en gris los resultados más destacados.

Fuente: Elaboración propia a partir de los resultados tabulados. 


\section{3. ¿Cómo valora el profesorado de la escuela rural su grado de desempeño?}

$\mathrm{Al}$ analizar las medias de las puntuaciones que valoran el grado de dominio de las competencias de la dimensión territorial, lo primero que se observa es que, a diferencia del caso anterior, las medias se sitúan claramente en un nivel medio (cuadro 2), en un rango comprendido entre los 2,61 y los 3,17 puntos.

También se observa una diferencia relevante con respecto a las puntuaciones que valoran su relevancia e importancia (figura 2). En líneas generales, esta diferencia para cada una de las competencias del cuestionario ( $\mathrm{M}$ dominio - $\mathrm{M}$ importancia) oscila en un rango que va de los $-0,40$ puntos a los $-0,80$ puntos.

Otro indicio que confirmaría esta percepción, se correspondería con una varianza considerable con respecto a la media de las puntaciones otorgadas por el profesorado a la hora de autocalificar su dominio de estas competencias. Salvo algunas excepciones que se comentarán a continuación, la mayoría de las medias presentan una varianza superior a los 0,50 puntos.

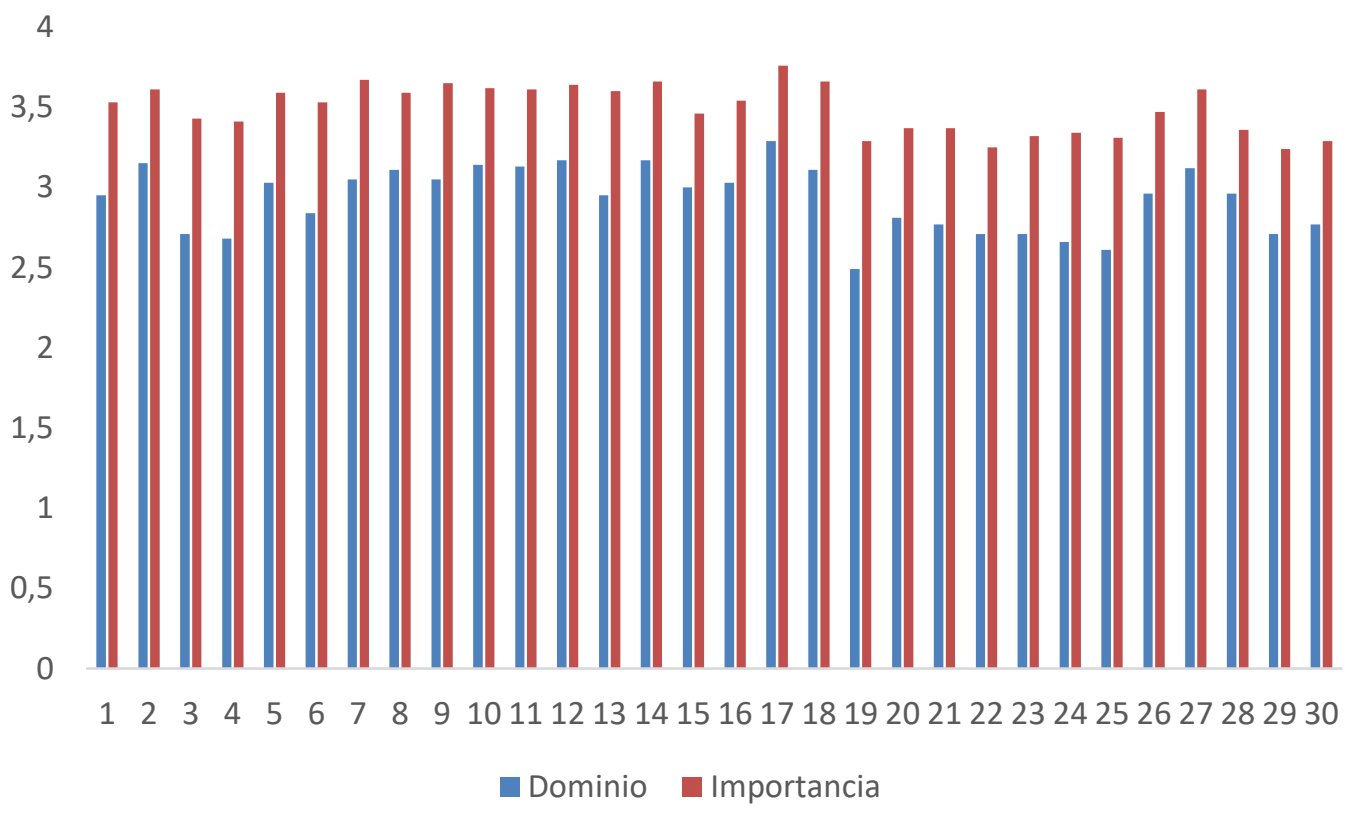

Figura 2. Valoraciones de dominio e importancia de las competencias de la Dimensión territorial

Fuente: Elaboración propia a partir de los resultados tabulados.

Por último, el análisis de los datos por cada uno de los ámbitos competenciales de la dimensión territorial no permite destacar lo siguiente.

\section{Ámbito pedagógico}

A pesar de las diferencias comentadas anteriormente, en este ámbito competencial se vuelve a observar un grado significativo de consistencia interna $(\alpha=0,87)$. Por otra parte, también se constata que tanto la competencia 2 (Incluir en la planificación docente las características culturales de su territorio rural, $\mathrm{M}=3,15, \mathrm{DE}=0,67$ ) como la competencia 5 (Implicar al equipo docente en los proyectos vinculados a su territorio rural, $\mathrm{M}=3,03$, $\mathrm{DE}=0,77)$ vuelven a ser las mejor valoradas cuando el profesorado evalúa su grado de 
desempeño. Este hecho, también se corrobora al constatar que, en comparación con el resto de competencias de este ámbito, son de las que muestran una menor varianza $\left(\sigma^{2}=\right.$ $0,45 \mathrm{y}^{2}=0,59$ respectivamente).

\section{Ámbito metodológico}

Las valoraciones acerca del grado de desempeño de las competencias relacionadas con este ámbito muestran la misma consistencia que la mostrada al valorar su relevancia $(\alpha=0,95)$.

No obstante, las puntuaciones medias obtenidas para cada una de las competencias son ostensiblemente más bajas que cuando se valora su importancia. En cualquier caso, salvo en el caso de la competencia 13 (que se valora menos) y 16 (que se valora más), las puntuaciones que superan los 3 puntos siguen correspondiendo con las competencias destacadas en el apartado anterior:

- C7: Sensibilizar al alumnado sobre las necesidades relacionados con el desarrollo sostenible de su territorio rural $(\mathrm{M}=3,05, \mathrm{DE}=0,78)$.

- C8: Desarrollar el conocimiento de la diversidad cultural de su territorio rural $(\mathrm{M}=3,11, \mathrm{DE}=0,73)$.

- C9: Desarrollar una actitud crítica ante los valores de su territorio rural $(\mathrm{M}=$ 3,05, DE $=0,76)$.

- C10: Tener en cuenta los conocimientos previos relacionados con la cultura de su territorio rural $(\mathrm{M}=3,14, \mathrm{DE}=0,71)$.

- C11: Establecer relaciones entre las experiencias previas vinculadas a su territorio rural y los nuevos aprendizajes $(\mathrm{M}=3,13, \mathrm{DE}=0,69)$.

- C12: Relacionar los contenidos con la realidad territorial inmediata $(\mathrm{M}=3,17$, $\mathrm{DE}=0,49)$.

- C14: Desarrollar actividades para el conocimiento de la cultura local ( $\mathrm{M}=3,17$, $\mathrm{DE}=0,72)$.

- C16: Considerar las características de su territorio rural en la organización de los espacios y tiempos escolares $(\mathrm{M}=3,03, \mathrm{DE}=0,80)$.

- C17: Utilizar los recursos que ofrece su territorio rural (naturales, materiales, sociales y culturales $)(\mathrm{M}=3,29, \mathrm{DE}=0,70)$.

- C18: Implicar en el centro a agentes de la comunidad local $(\mathrm{M}=3,11, \mathrm{DE}=$ $0,77)$.

Por otra parte, el hecho de que la varianza al puntuar estas competencias sea mucho más elevada que cuando se valora su importancia $\left(\sigma^{2}=\right.$ entre 0,48 y 0,65$)$, aun y ser de las más bajas de la serie, permite percibir una cierta dispersión en estas valoraciones.

\section{Ámbito relación comunidad-escuela}

Finalmente, en lo que respecta a las competencias vinculadas con este ámbito destacar también su elevado grado de consistencia interna $(\alpha=0,89)$. Asimismo, las puntuaciones más elevadas también se han correspondido con las competencias mejor valoradas en cuanto a su relevancia. Sin embargo, en este caso, la competencia con mayor puntuación ha sido la competencia 27 (Establecer procesos de participación escuela-familiascomunidad y viceversa, $\mathrm{M}=3,12, \mathrm{DE}=0,80)$; y además de la competencia 26 
(Comprometerse con iniciativas socio-culturales de la comunidad, $\mathrm{M}=2,96, \mathrm{DE}=0,77$ ) debe de añadirse también la competencia 28 como la más valorada (Implicar a la familia en actividades extraescolares, $\mathrm{M}=2,96, \mathrm{DE}=0,82$ ).

$\mathrm{Al}$ igual en los casos anteriores, el hecho que la varianza de las medias registradas en estas tres no sea muy elevada confirmaría la relevancia de estas valoraciones (competencia 27, $\sigma^{2}=0,59$; competencia $26, \sigma^{2}=0,64 ;$ y competencia $\left.28, \sigma^{2}=0,68\right)$.

\section{Discusión y conclusiones}

Actualmente el medio rural está viviendo numerosos cambios de índole social y económica los cuales, a su vez, inciden en las relaciones que se establecen, tanto entre los miembros de sus comunidades escolares, como entre éstas últimas y las administraciones locales.

Para dar respuesta a este fenómeno, las escuelas rurales requieren de un profesorado especializado que, además de dominar las competencias docentes básicas, también muestre su competencia a la hora de incorporar en sus propuestas didácticas las necesidades formativas especificas del alumnado de la escuela rural; la conciencia y la preservación de la identidad del territorio al cual pertenecen; el conocimiento y la preservación de sus costumbres, tradiciones y patrimonio. Al respecto, y teniendo en cuenta los objetivos del estudio aquí presentados, los resultados obtenidos nos permiten considerar lo siguiente.

En lo referente al perfil del profesorado que debe desempeñar estas competencias relacionadas con la dimensión territorial, el profesorado encuestado, dispone de una situación laboral, antigüedad en la escuela y expectativas profesionales que, en la línea de lo expresado por los organismos europeos (Comisión Europea, 2005, 2007), Mulder, Weigel y Collings (2008) o Aguerrondo (2009), podría facilitar la implementación de proyectos educativos y propuestas didácticas que tuvieran la ruralidad como centro de interés. No obstante, es evidente que para valorar el impacto real de esta aseveración no puede obviarse aquí que, a juzgar por los resultados, la formación especializada que ha recibido el profesorado encuestado ha sido escasa, y que ésta se ha realizado de forma predominante en el ámbito de la formación continuada. Asimismo, tampoco puede obviarse el hecho que en este estudio no se ha obtenido información concreta con respecto al tipo de formación recibida.

En lo que respecta a los contextos en donde el profesorado está ejerciendo su labor docente, destacar que, si se parte de la base que las escuelas situadas en municipios pequeños suelen tener una vinculación muy estrecha con el territorio al cual éstas pertenecen, este dato podría también ser de mucha utilidad para determinar si el desempeño de las competencias relacionadas con la dimensión territorial tendría más posibilidades de éxito en este tipo de contextos.

Por otra parte, a la hora de valorar las competencias profesionales que el profesorado considera más necesarias para el desempeño de la dimensión territorial, en primer lugar, puede decirse que las competencias identificadas en el marco de este estudio se han mostrado pertinentes y relevantes. Este hecho se deduce de los elevados índices de coherencia interna obtenidos tras analizar las respuestas de los maestros y maestras rurales. Asimismo, estos resultados también confirmarían la pertinencia de los marcos de referencia que se tuvieron en cuenta para la confección del catálogo de competencias (Comisión Europea, 2004, 2007; González y Wagenaar, 2010; Perrenaud, 2007). 
En esta misma línea, el análisis de los resultados también ha permitido observar que estas competencias han sido valoradas por los maestros rurales encuestados como necesarias y relevantes para la formación específica de estos docentes. Este hecho está en plena concordancia con lo expresado por la Comisión Europea (2005, 2007) a la hora de identificar y definir las competencias y procesos necesarios para una formación del profesorado de calidad, capaz de ejercer su función docente y social en cualquier contexto educativo.

En cuanto al balance que el profesorado realiza con respecto al grado de dominio de estas competencias, por norma general, se ha observado que el profesorado de la escuela rural suele valorar el dominio de determinadas competencias con puntuaciones más bajas, en comparación con las valoraciones realizadas acerca de su grado de importancia.

Estas diferencias, permitirían identificar una serie de necesidades y prioridades formativas que deberían de ser tenidas en cuenta a la hora de diseñar cualquier tipo de plan específico de formación destinado a la capacitación del profesorado de la escuela rural y, por extensión, con el desempeño de las competencias que desarrollan la dimensión territorial. Sobre todo, si se tiene en cuenta la recomendación de la Comisión Europea (2007, 2014) cuando sugiere que las propuestas formativas centradas en el dominio de competencias docentes deberán de adaptarse a las características del contexto, de los enseñantes y de las necesidades formativas de cada estado miembro. En este sentido, una futura línea de investigación se correspondería con el hecho de aprovechar los resultados obtenidos de este balance de competencias para poder identificar cuáles deberían ser las competencias y los contenidos clave sobre las cuales llevar a cabo estas propuestas de formación específica, tanto en el marco de la formación inicial como continuada.

Como conclusión final, los resultados obtenidos permiten esgrimir la idea que el profesorado de la escuela rural catalana considera que el grado de dominio de sus competencias relacionadas con la dimensión territorial no se suele corresponder con lo esperado con respecto a su relevancia o importancia. Esta circunstancia podría explicarse por la falta de formación específica recibida con respecto a cómo desempeñar en el alumnado las competencias vinculadas a la dimensión territorial. En este sentido, no puede olvidarse que, en el ámbito de la escuela rural, es muy frecuente trabajar con un alto grado de intuición y voluntarismo por parte del profesorado, sin tener en cuenta que es necesaria una formación contextualizada que, por ejemplo, permita tanto la puesta en práctica de acciones didácticas específicas y pertinentes, como el establecimiento de vínculos clave con diversos agentes educativos del territorio. Por este motivo, sería también recomendable realizar estudios de corte ideográfico a partir de los cuales fuese posible llegar a establecer modelos y pautas de actuación que ayudasen al profesorado a desempeñar tanto su labor docente como a establecer y consolidar las relaciones pertinentes con las personas y los entes que forman parte de un determinado territorio o contexto rural.

\section{Agradecimientos}

Este estudio forma parte del proyecto "La Formación profesional y las competencias del maestro rural como dinamizador de la dimensión territorial en la escuela" (2017-1-ESo1KA201-038217), subvencionado por la Unión Europea en el marco del programa Erasmus+ 2017 (Acción KA201-Asociacones estratégicas). 


\section{Referencias}

Abós, P. (2011). La escuela en el medio rural y su presencia en los planes de estudio de los grados de maestro en educación infantil y primaria de las universidades españolas. Profesorado. Revista de Currículum y Formación del Profesorado, 15(2), 39-52.

Abós, P. (2015). El modelo de escuela rural ¿Es un modelo transferible a otro tipo de escuela? Educação E̊ Realidade, 4O(3), 667-684. https://doi.org/10.1590/2175-623645781

Aguerrondo, I. (2009). Complex knowledge and education competences. Geneve: UNESCO-IBE.

Arriaga, N. y Ruiz-Gallardo, J. (2017). Colegios rurales agrupados y formación universitaria. Profesorado. Revista de Currículum y Formación de Profesorado, 21(4), 215-240.

Boix, R. (2014). La escuela rural en la dimensión territorial. Innovación Educativa, 24, 89-97.

Buscà, F., Domingo, L. y Boix R. (2018). Incidència de la metodologia en l'avaluació de les competències bàsiques a l'escola rural: El cas de la competència lingüística. Didácticae. Revista de Investigación en Didácticas Específicas, 4, 108-125.

Bustos, A. (2011). Investigación y escuela rural ¿Irreconciliables? Profesorado. Revista de Curriculum y Formación del Profesorado, 15(2), 155-170.

Cornish, L. (2006). Multi-age practices and multi-grade classes. En L. Cornish (Ed.), Reaching EFA through multi-grade teaching (pp. 27-48). Armidale: Kardoorair Press.

Chaparro-Aguado, F. y Santos-Pastor, M. L. (2018). Competencias docentes para la escuela rural en la formación inicial. Análisis de resultados de un estudio multicaso Education, Sport, Health and Physical Activity, 2(2),177-191.

Comisión Europea. (2004). Competencias clave para un aprendizaje a lo largo de la vida. Un marco de referencia europeo. Luxemburgo: Oficina de Publicaciones de la Unión Europea.

Comisión Europea. (2005). Common European principles for teacher competences and qualifications. Luxemburgo: Oficina de Publicaciones de la Unión Europea.

Comisión Europea. (2007). Improving the quality of teacher education. Luxemburgo: Oficina de Publicaciones de la Unión Europea.

Comisión Europea. (2014). Horizonte 2020 en breve. Luxemburgo: Oficina de Publicaciones de la Unión Europea.

Domingo, L. (2014). Contribucions pedagògiques de l'escola rural. La inclusió a les aules multigrau: Un estudi de cas (Tesis doctoral). Universitat de Vic.

González, J. y Wagenaar, R. (2010). Tuning educational structures in Europe. Tuning educational structures in Europe. https://doi.org/10.1163/157007490X00133

McMillan, J. H. y Shumacher, S. (2011). Investigación educativa. Madrid: Pearson-Addison Wesley.

Mulder, M., Weigel, T. y Collings, K. (2008). El concepto de competencia en el desarrollo de la educación y formación profesional en algunos estados miembros de la UE: Un análisis crítico. Profesorado. Revista de Currículum y Formación del Profesorado, 12(3), 1-24.

Perrenaud, P. (2007). Diez nuevas competencias para enseñar. Barcelona: Graó.

Raso, F., Hinojo, M. A. y Solá, J. M. (2015). Integración y uso docente de las tecnologías de la información y la comunicación (TIC) en la escuela rural de la provincia de Granada: Estudio descriptivo. REICE. Revista Iberoamericana sobre Calidad, Eficacia y Cambio en Educación, 13(1), 139-159. 
Rubio, P. (2010). Modelización de los cambios y evolución reciente del sistema rural español. Boletín de la Asociación de Geógrafos Españoles, 54, 203-235.

Serret, M. y Trepat, E. (2009). Atles de la nova ruralitat. Lleida: Fundació del Món Rural.

Solé, M. (2016). Escola i territori: Les ZER a Catalunya. Revista Catalana de Pedagogía, 10, 75-93.

Trepat, E. (2015). Atles de la nova ruralitat. L'actualitat del món rural. Els anys dela gran crisis a la Catalunya rural, 2008-2015. Lleida: Fundació del Món Rural. 


\section{Anexo. Ítems del cuestionario de competencias docentes relacionadas con la dimensión territorial}

\begin{tabular}{|c|c|c|}
\hline ÁмвIто & ÍTEM & COMPETENCIA \\
\hline \multirow{6}{*}{ Pedagógico } & 1 & $\begin{array}{l}\text { Considerar las necesidades de su territorio rural en la planificación } \\
\text { docente }\end{array}$ \\
\hline & 2 & $\begin{array}{l}\text { Incluir en la planificación docente las características culturales de } \\
\text { su territorio rural }\end{array}$ \\
\hline & 3 & $\begin{array}{l}\text { Identificar las necesidades formativas del profesorado del centro en } \\
\text { relación con su territorio rural }\end{array}$ \\
\hline & 4 & $\begin{array}{l}\text { Posibilitar acciones de desarrollo profesional para el profesorado } \\
\text { del centro en relación con las necesidades formativas detectadas }\end{array}$ \\
\hline & 5 & $\begin{array}{l}\text { Implicar al equipo docente en los proyectos vinculados a su } \\
\text { territorio rural }\end{array}$ \\
\hline & 6 & $\begin{array}{l}\text { Incluir las necesidades de su territorio rural en el diseño de los } \\
\text { proyectos de innovación. }\end{array}$ \\
\hline \multirow{17}{*}{ Metodológico } & 7 & $\begin{array}{l}\text { Sensibilizar al alumnado sobre las necesidades relacionados con el } \\
\text { desarrollo sostenible de su territorio rural }\end{array}$ \\
\hline & 8 & $\begin{array}{l}\text { Desarrollar el conocimiento de la diversidad cultural de su } \\
\text { territorio rural }\end{array}$ \\
\hline & 9 & Desarrollar una actitud crítica ante los valores de su territorio rural \\
\hline & 10 & $\begin{array}{l}\text { Tener en cuenta los conocimientos previos relacionados con la } \\
\text { cultura de su territorio rural }\end{array}$ \\
\hline & 11 & $\begin{array}{l}\text { Establecer relaciones entre las experiencias previas vinculadas a su } \\
\text { territorio rural y los nuevos aprendizajes }\end{array}$ \\
\hline & 12 & Relacionar los contenidos con la realidad territorial inmediata \\
\hline & 13 & $\begin{array}{l}\text { Proponer situaciones de investigación relacionadas con el entorno } \\
\text { rural próximo }\end{array}$ \\
\hline & 14 & Desarrollar actividades para el conocimiento de la cultura local \\
\hline & 15 & $\begin{array}{l}\text { Realizar actividades complementarias que partan del territorio } \\
\text { rural }\end{array}$ \\
\hline & 16 & $\begin{array}{l}\text { Considerar las características de su territorio rural en la } \\
\text { organización de los espacios y tiempos escolares }\end{array}$ \\
\hline & 17 & $\begin{array}{l}\text { Utilizar los recursos que ofrece su territorio rural (naturales, } \\
\text { materiales, sociales y culturales) }\end{array}$ \\
\hline & 18 & Implicar en el centro a agentes de la comunidad local \\
\hline & 19 & $\begin{array}{l}\text { Elaborar materiales curriculares con participación de agentes de su } \\
\text { territorio rural }\end{array}$ \\
\hline & 20 & $\begin{array}{l}\text { Incorporar el uso de TIC para el conocimiento de territorio rural } \\
\text { próximo }\end{array}$ \\
\hline & 21 & $\begin{array}{l}\text { Utilizar las TIC como elemento de comunicación con los agentes } \\
\text { de su territorio rural }\end{array}$ \\
\hline & 22 & $\begin{array}{l}\text { Considerar los saberes sobre la cultura local en la evaluación de los } \\
\text { aprendizajes. }\end{array}$ \\
\hline & 23 & $\begin{array}{l}\text { Considerar los contenidos relacionados con el desarrollo sostenible } \\
\text { de su territorio rural en la evaluación de los aprendizajes }\end{array}$ \\
\hline \multirow{4}{*}{$\begin{array}{l}\text { Relación } \\
\text { escuela- } \\
\text { comunidad }\end{array}$} & 24 & $\begin{array}{l}\text { Implicar a la comunidad local en el diseño, desarrollo y evaluación } \\
\text { del proyecto educativo de centro incorporando sus expectativas }\end{array}$ \\
\hline & 25 & $\begin{array}{l}\text { Incluir en los objetivos de la escuela el servicio al desarrollo } \\
\text { cultural de su territorio rural. }\end{array}$ \\
\hline & 26 & Comprometerse con iniciativas socio-culturales de la comunidad \\
\hline & 27 & $\begin{array}{l}\text { Establecer procesos de participación escuela-familias-comunidad y } \\
\text { viceversa }\end{array}$ \\
\hline
\end{tabular}




\begin{tabular}{ll}
\hline 28 & Implicar a la familia en actividades extraescolares \\
29 & Implicar a la comunidad local en actividades extraescolares \\
30 & Utilizar la escuela como espacio cultural de la comunidad local \\
\hline
\end{tabular}
Fuente: Elaboración propia a partir de la validación del cuestionario.

\section{Breve CV de los autores}

\section{Roser Boix}

Doctora por la Universitat de Barcelona (España) y Licenciada en Ciencias de la Educación por la Universitat de Barcelona. Actualmente ejerce como Profesora Titular de Universidad en la Facultad de Educación (Universitat de Barcelona) desempeñando al mismo tiempo el cargo de Decana de la Facultad. Miembro del Observatorio de Educación Rural de Cataluña, del Grupo Interuniversitario de Escuela Rural y del Secretariado de Escuela Rural de Cataluña. Sus líneas de investigación se sitúan en torno a la escuela multigrado, la educación rural y la formación inicial y permanente del maestro rural. ORCID ID: https://orcid.org/oooo-001-9000-7967. Email: roser.boix@ub.edu

\section{Francesc Buscà}

Doctor por la Universitat de Barcelona (España) y Licenciado en educación física por el INEFC-Universidad de Lleida (España). Actualmente ejerce como profesor agregado en la Facultad de Educación (Universitat de Barcelona), desempeñando al mismo tiempo el cargo de director del departamento de Didácticas Aplicadas. Miembro del GISEAFE (Grup d'investigació Social i Educativa de l'Activitat Física i l'Esport (2017-SGR1162) del INEFC-Universidad de Barcelona. Sus líneas principales de investigación se sitúan en torno a los siguientes ámbitos: desempeño de las competencias clave a través de la educación física escolar; la formación y capacitación de su profesorado en contextos educativos formales y no formales. ORCID ID: https://orcid.org/0000-0001-8831-8379. Email: fbusca@ub.edu 